\title{
LA SCHIZOPHRENIE
}

\section{Mieux comprendre la maladie}

\section{Radia Hadjlakehal}

Faculté des sciences humaines et sociales

\section{Université de Biskra algerie}

\section{Résumé:}

La schizophrénie est une maladie complexe, elle est difficile à comprendre, difficile à soigner, difficile à vivre, et comme tout ce que nous avons du mal à comprendre, elle fait peur.

A travers cet article on essayera de donner beaucoup d'informations sur la maladie. Même si elles sont loin d'être complètes et parfaitement organisées, les connaissances permettent souvent de faire tomber les peurs, les idées fausses et les préjugés.

يعتبر مرض الفصام من اعقد الأمراض النفسية، نظرا لصعوبة فهمه

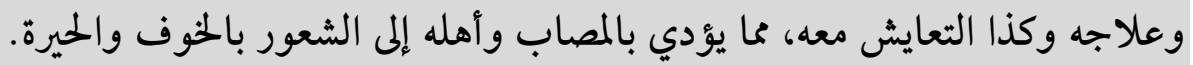
يحاول هذا المقال رفع الستار حول الاضطراب ومدى تعقيده حتى يصحح الأفكار الخاطئة والمخاوف المتتشرة حوله. 


\section{Introduction:}

La schizophrénie, comme d'autres maladies psychiques, demeure méconnue et fortement stigmatisée dans notre société d'aujourd'hui. Ainsi, lorsque les premiers symptômes de ce trouble apparaissent chez un individu, ce sont encore trop souvent l'incompréhension, le rejet ou l'indifférence qui predominant ${ }^{(1)}$.

Schizophrénie, schizophrène, le mot a été forgé en 1911 par le psychiatre suisse Eugen Bleuler pour l'étymologie signifie « rupture de la pensée »(du grec skhizein, fissure, division, et phren, pensée).

Il souhaitait alors caractériser la rupture du fonctionnement psychique qu'il observait chez ses patients. Contrairement à l'idée répandue, le terme de schizophrénie n'a pas été crée pour décrire un dédoublement de la personnalité. Il désigne surtout un des symptômes fréquents dans cette maladie, la dissociation, qui peut être définie comme une perte de la cohésion de la pensée. Cette dernière est embrouillée, désordonnée ${ }^{(2)}$.

Il y a plus d'un siècle Emil Kraeplin avait cru que ces malades étaient des déments précoces, caractérisés par la perte de la raison, des émotions, des affectes. En 1911 son élève Eugene Bleuler sut relever que derrière la désorganisation psycho-comportemental existe une riche vie intellectuelle et affective. Le schizophrène est capable de sentir, de réfléchir et d'aimer ${ }^{(3)}$. 
L'entrée dans la maladie se fait essentiellement à l'adolescence ou au début de l'âge adulte et les formes inaugurales ont de multiples visages. Ainsi, ce sont parfois des états dépressifs, des troubles du comportement alimentaire, un fléchissement scolaire ou encore des épisodes de violence physique qui annoncent le début de la maladie.

Pour la plupart des gens, la schizophrénie, c'est le dédoublement de la personnalité, mais il faut dire tout de suite, la schizophrénie ce n'est pas le dédoublement de la personnalité(4).

Dans cet article on présente comment peut se manifester cette maladie et d'où peuvent provenir les symptômes qui la composent et les causes et les mécanismes de la schizophrénie. En les présentant en détail.

\section{1-Qu'est ce que la schizophrénie?}

A l'heure actuelle, la schizophrénie demeure l'une des maladies mal connues et taboues de notre époque.

La schizophrénie est ainsi considérée comme plus abstraite ou plus « mystérieuse » que d'autre maladies chroniques. Pourtant, parmi toutes les sources de souffrance, l'atteinte de l'intégrité psychique est probablement de celles qui entravent le plus le fonctionnement de l'individu, tant elle touche à ce qu'il a de plus intime. Le taux de suicide très élevés chez les individus atteints de schizophrénie témoigne également de l'intensité de leur souffrance ${ }^{(5)}$. 
Elle se distingue des autres psychoses maniaco-dépressives ou troubles bipolaires, dont les accès maniaques peuvent prendre l'allure d'épisodes schizophréniques aigus, mais qui évoluent par poussées entrecoupées de périodes libres de tout symptôme, et par la coexistence de plusieurs symptômes (contrairement au trouble délirant ou paranoïa, ou le délire s'exprime de manière généralement isolée).

Les patients souffrant de schizophrénie sont confrontés à des expériences particulièrement troublantes: ils peuvent entendre des voix s'adressant à eux de manière blessante, alors même qu'ils sont seuls (ces manifestations sont appelées hallucinations accousticoverbales) et ils ont aussi, fréquemment, l'impression de perdre le contrôle d'une partie de leurs pensées et de leurs actions, qu'ils vivent parfois comme leur étant imposées de l'extérieur ( ce phénomène, souvent associé aux hallucinations verbales, est appelé syndrome d'influence) ${ }^{(6)}$.

\section{La difficulté du diagnostic (les principaux symptômes)}

Le diagnostic de schizophrénie repose sur un ensemble de symptômes d'évolution chronique. Il n'y a aucun symptôme spécifique, et c'est l'association de plusieurs éléments qui permet de porter ce diagnostic. Le facteur temps est un indice capital, car l'évolution fait parfois reconsidérer le diagnostic de la maladie. En effet il n'existe à ce jour aucun examen de laboratoire ou d'imagerie cérébrale permettant d'affirmer ou d'éliminer ce diagnostic. 
Dans la conception actuelle de la schizophrénie, les symptômes sont classés selon deux groupes principaux :

1-Les symptômes positifs : représentent souvent les symptômes qui apparaissent comme «produits» par la maladie, en particulier les hallucinations et les idées délirantes, un discours ou des comportements désorganisés, cela reste pourtant des symptômes et le terme de positif ne veut pas dire qu'ils soient appréciables ou souhaitables. Le plus souvent, ils correspondent au fait d'entendre une ou plusieurs voix et à avoir des convictions et des croyances étranges.

\section{1-1-Les hallucinations :}

Les hallucinations peuvent revêtir plusieurs formes : une voix qui parle dans la tête, à l'extérieur de la tête, du même sexe ou de sexe différent de la personne qui l'entend, parfois même les voix peuvent être multiples et se répandre. Elles peuvent également consister en des bruits ou de la musique, des chuchotements incompréhensibles. Le plus souvent, les hallucinations auditives prennent la forme de propos agressifs, les patients peuvent entendre des insultes, des critiques ou encore des menaces, quelquefois, ces voix sont moins désagréables et peuvent consister en des conseils ou des avertissements.

Bien que les hallucinations auditives soient les plus fréquentes, il arrive également qu'elles soient tactiles. On parle alors d'hallucinations cénesthésiques. Ce sont des sensations de toucher: une main passée dans les cheveux, des sensations de contact des zones génitales, des pressions inexpliquées dans le ventre. 
Enfin, des hallucinations visuelles apparaissent parfois également. Plus rarement, des odeurs ou des goûts étranges sont perçus.

On peut dire que lors des hallucinations auditives ou visuelles le malade entend alors des voix qui le tourmentent, le font souffrir, peuvent l'accuser, lui donner des ordres, le contrôler, voire le menacer, parfois, il voit des êtres venus d'un autre monde ou d'une autre époque pour l'agresser.

\section{1-2-Les convictions délirantes}

Un autre symptôme de la schizophrénie est d'être convaincu de choses qui peuvent paraître étranges aux autres. Un délire est une fausse croyance maintenue avec une certitude absolue en dépit de la preuve du contraire, et étrangère au cadre social, culturel, éducationnel et religieux de la personne.

Les idées délirantes peuvent correspondre à des convictions pour des choses impossibles par exemple : « un animal vit dans ma tête » « je peux changer le climat ».Il y a aussi beaucoup de croyances non scientifiques tenues pour vraies dans une large proportion de la population par exemple, croyances dans la télépathie, les esprits frappes, enlèvement par des extra-terrestre, horoscopes qui se confondent avec les croyances «délirantes » et il n'y a aucun élément distinctif pour lequel elles ne sont pas en accord avec l'arrière plan culturel de la personne. 
On conclut que le délire est une modification du raisonnement et qui conduit à une fausse conviction. Le schizophrène est plongé dans un monde inquiétant et angoissant; très souvent, l'individu est convaincu qu'il est persécuté, en danger, qu'on veut le tuer, qu'il est responsable des malheurs dans le monde ou investi d'une mission que lui seul comprend ${ }^{(7)}$.

\section{2-Les symptômes négatifs :}

Contrairement aux symptômes positifs, à la fois manifestes et inquiétants, mais ne compromettant pas nécessairement l'activité des personnes qui en souffrent et généralement sensibles au traitement, les symptômes négatifs sont beaucoup plus insidieux, durables et difficiles à traiter. Ils sont de ce fait également très invalidants. Ces caractéristiques, associées à leur fréquence au sein de la population des schizophrènes, en font un défi majeur pour la recherche dans le domaine du traitement de la schizophrénie. Par ailleurs, il est fréquent qu'ils soient associés à des symptômes positifs (par exemple un délire) peu bruyants, mais parfois très riches ${ }^{(8)}$.

\section{2-1-Emoussement affectif}

Les symptômes négatifs peuvent être plus discrets même s'ils sont tout aussi invalidants. Les émotions peuvent devenir abrasées, on observe alors un émoussement affectif, c'est-à-dire une diminution de l'expression et du ressenti des émotions positifs et négatives, joie ou tristesse disparaissent. Les soignants parlent parfois de $«$ froideur ${ }^{(9)}$. 
L'émoussement affectif peut être une réaction directe à des voix ou à des pensées agressives ou attentatoires, et l'expression «figée », une «façade» vis-à-vis du monde, peut être une tentative pour faire face à une perturbation qui paraît écrasante. Ces symptômes se manifestent de diverses manières souvent peu marquées. Mais la diminution de l'expression émotionnelle et particulièrement bien reconnue comme effet secondaire ${ }^{(10)}$.

\section{2-2-Le ralentissement de la pensée}

Un autre type de symptôme négatif touche, quant à lui, les fonctions dites cognitives, c'est-à-dire les activités de la pensée. On repère notamment un ralentissement de la pensée et de la réflexion. Une diminution dans la production langagière ainsi qu'une perte de volonté pour accomplir n'importe quelle activité peuvent également faire partie des manifestations problématiques.

La perte de volonté atteint jusqu'à des activités se rapportant à soi, à négliger son alimentation ou son hygiène personnelle. Il arrive aussi que les patients soient indifférents aux contacts sociaux, se replient sur eux-mêmes, au risque d'échouer dans une complète immobilité ou dans un mutisme (catatonie) $)^{(11)}$.

Les symptômes négatifs correspondent à ce qui est altéré ou diminué par la maladie comme la capacité à entretenir des relations sociales et affectives, à travailler, à s'occuper de soi et à gérer les situations du quotidien. 


\section{3-La désorganisation de la pensée :}

D'autres symptômes sont relatifs à la désorganisation de la pensée dont souffrent parfois certains malades. Ce relâchement des associations entre les thèmes de réflexion donne souvent une impression générale de confusion qui correspond parfois à celle que peut vivre le malade. On peut observer des coq-à-l'âne, des changements abrupts des thèmes de la conversation sans lien logique entre eux ${ }^{(12)}$.

Il arrive également que certains dérèglements émotionnels conduisent les malades à ne plus contrôler leurs émotions, ce qui peut les faire rire ou pleurer sans en connaître la raison ou dans des situations qui déclenchent habituellement des émotions différentes ${ }^{(13)}$ donc la perturbation de la logique de la pensée, qui se manifeste par un langage incompréhensible, avec des associations d'idées décousues.

Tableau 1 : Les trois principales dimensions symptomatiques de la Schizophrénie ${ }^{(14)}$

\begin{tabular}{|c|r|}
\hline Dimension & Description \\
\hline Positive & $\begin{array}{r}\text { Modification du vécu (perturbation de la relation } \\
\text { à l'environnement et à son propre corps- } \\
\text { hallucinations et délire) }\end{array}$ \\
\hline Négative & Incapacité à agir ou éprouver \\
\hline Désorganisation & $\begin{array}{r}\text { Incapacité à construire son comportement et son } \\
\text { discours selon un plan structuré }\end{array}$ \\
\hline
\end{tabular}




\section{Tableau 2 : Les principaux symptômes positifs de la}

Schizophrénie ${ }^{(15)}$

\begin{tabular}{|r|r|}
\hline \multicolumn{1}{|c|}{ symptôme } & \multicolumn{1}{|c|}{ Description } \\
\hline $\begin{array}{r}\text { Hallucination } \\
\text { verbales }\end{array}$ & Audition de voix alors que personne ne s'exprime \\
\hline $\begin{array}{r}\text { Hallucinations } \\
\text { cinesthésique }\end{array}$ & sensations corporelles profondes sans support \\
organique \\
\hline $\begin{array}{r}\text { Syndrome } \\
\text { d'influence }\end{array}$ & Sensation de contrôle de ses actions par une force \\
\hline extérieure \\
\hline Pensées imposées & Pensées placées dans sa tête par une force \\
\hline Diffusion de la & \\
\hline pensée & Impression que les autres lisent les pensées \\
\hline Vol de la pensée & Impression que les autres dérobent des pensées \\
\hline Idées délirantes & Convictions non fondées sur des faits réels \\
\hline
\end{tabular}

Tableau 3 : Les principaux symptômes négatifs de la Schizophrénie $^{(16)}$ :

\begin{tabular}{|r|r|}
\hline \multicolumn{1}{|c|}{ symptômes } & Description \\
\hline Aboulie & Affaiblissement de la volonté \\
\hline Apragmatisme & Incapacité à entreprendre des actions \\
\hline
\end{tabular}




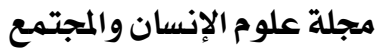

\begin{tabular}{|r|r|}
\hline Anhédonie & Incapacité à ressentir du plaisir \\
\hline Energie physique & Manque d'énergie, absence d'initiative \\
\hline Incurie & Impossibilité de prendre soin de soi \\
\hline
\end{tabular}

Tableau 4 : La désorganisation Schizophrénique ${ }^{(17)}$ :

\begin{tabular}{|r|r|}
\hline \multicolumn{1}{|c|}{ Symptômes } & \multicolumn{1}{|c|}{ Description } \\
\hline Incohérence motrice & Activité désordonnée \\
\hline Incohérence du discours & Langage peu ou non compréhensible \\
\hline Paralogismes, néologismes & Emploi de termes inappropriés ou \\
inventés
\end{tabular}

Le diagnostic est porté devant la coexistence d'un faisceau de symptômes sur une durée de plusieurs mois, dont la présence entraîne une souffrance importante pour le patient et pour son entourage, entravant son niveau de fonctionnement psycho-social et professionnel.

Les classifications internationales des maladies (classification de I'OMS et classification nord-américaine DSM) proposent des regroupements qui nécessitent la présence d'un certain nombre de symptômes sur une période de temps suffisante pour aboutir à une probabilité statistique importante de faire un diagnostic correct. Ces classifications, si elles ont permis d'homogénéiser les critères de la 
maladie et d'aplanir les « querelles d'écoles » sont surtout utiles pour la recherche.

Ainsi le diagnostic de trouble schizophrénique nécessite l'expertise d'un médecin spécialisé en psychiatrie, qui permettra non seulement de le confirmer ou de l'infirmer, mais aussi d'éliminer une pathologie médicale sous-jacente (hormonale, neurologique, toxique.... ${ }^{(18)}$.

\section{La fréquence des schizophrénies : (épidémiologie)}

La schizophrénie touche environ $1 \%$ de la population, elle est présente dans tous les pays du monde et dans tous les milieux sociaux. En moyenne, la schizophrénie apparaît entre 15 et 25 ans, avec, d'après les études épidémiologiques, un début souvent plus précoce pour les garçons que pour les filles, par ailleurs, il existe, bien plus rarement, des formes à début tardif, qui débutent vers la cinquantaine, et semblent plus fréquentes chez les femmes ${ }^{(19)}$.

Il arrive parfois que la maladie se déclenche au cours de l'adolescence. Son apparition est souvent progressive et insidieuse. Elle se manifeste généralement au travers de signes non spécifique, c'est-à-dire que les symptômes ne sont pas présents d'emblée. Une perte d'intérêt pour le travail ou l'école peut être par exemple observée, un besoin de plus en plus marqué de se retrouver seul et de se mettre en retrait des relations sociales, ou encore des accès de colère, de légers problèmes de mémoire ou d'attention, une détérioration de l'hygiène etc... ${ }^{(20)}$. 
La schizophrénie est une maladie des sujets jeunes, elle handicape gravement leur développement et leur épanouissement. Elle survient sous l'influence de facteurs déclenchants biologiques et psychologiques chez des sujets porteurs d'une vulnérabilité, elle aussi biologique ou psychologique ${ }^{(21)}$.

\section{Les causes de la schizophrénie}

Qu'elles sont les causes de la schizophrénie ?

La question des causes de la schizophrénie ne peut être envisagée de manière simple et univoque, en effet, ce trouble ne répond certainement pas à une cause unique, mais à un ensemble de facteurs (en particulier génétiques et environnementaux précoces) se combinant pour réaliser ce que l'on appelle une vulnérabilité, cela signifie concrètement que, d'une part, les personnes souffrant de schizophrénie ne sont pas nées malades, mais qu'elles présentaient d'emblée une plus grande susceptibilité que les autres de développer cette maladie et que, d'autre part, pour s'exprimer, cette fragilité doit parfois être révélée par l'intervention de facteurs stressants de divers ordres (traumatismes, conflits, etc...). Plus la fragilité est importante, moins l'intervention de facteurs stressants sera nécessaire pour provoquer l'entrée dans la maladie ${ }^{(22)}$.

Une cause unique est à peu prés inexistante puisqu'elle engendre immédiatement et nécessairement une cascade d'interactions avec les autres éléments du système, de sorte que l'effet final sera constitué par la somme de toutes ces interactions ${ }^{(23)}$. 
Certains des symptômes de la schizophrénie peuvent être retrouvés chez nombre d'entre nous qui ne souffrent pas de cette maladie. Les idées étranges, les hallucinations, la déréalisation, la dépersonnalisation peuvent apparaitre à des moments forts de notre vie, si chacun peut rencontrer ces phénomènes, pourquoi certaines personnes développent cette maladie et pas d'autres ? Une fois encore, il n'y a pas une seule raison, mais plusieurs facteurs qui permettraient de répondre à cette question.

\section{1-Les causes génétiques}

De nombreuses recherches se sont penchées sur l'implication des gènes dans la schizophrénie. Il est vrai que certaines données peuvent conduire à penser que la maladie se transmet génétiquement, alors que la fréquence d'apparition est de 1\%. Généralement, elle oscille entre 5 et $8 \%$ lorsqu'un parent est déjà touché. Quand les deux parents souffrent de schizophrénie, le pourcentage de risque que leur enfant déclare la maladie est de $40 \%$. Enfin, chez les vrais jumeaux, quand l'un des deux est touché par la maladie, il y a 50\% de risque que l'autre le soit également.

Ces résultats peuvent conduire à penser qu'une part des causes de la schizophrénie repose sur les gènes. Mais une part seulement. Chez les vrais jumeaux qui partagent exactement le même patrimoine génétique, le taux ne passe pas en effet à 100\% comme on devrait l'observer si les gènes étaient totalement responsables de la maladie. 
Il existe une composante héréditaire dans la schizophrénie, mais pour autant, il ne s'agit pas d'une maladie purement génétique. La schizophrénie est une maladie où de multiples facteurs sont en cause et les facteurs génétiques semblent interagir étroitement avec différents facteur liés à l'environnement et dont la conjonction détermine une vulnérabilité. C'est une maladie multifactorielle. 


\section{Références:}

(1) Gourion David et Gut-Fayand Anne, Les troubles schizophréniques, vivre et comprendre, Ellipses Editions marketing.S.A Paris.2004,P.9.

(2) Bourgeois. Marc Louis, Les schizophrénies, Que sais-je ?, $6^{\mathrm{eme}}$ Editions mise à jour. PWF, Paris, 2011. P13.

(3) Granger Bernard, Naudir Jean, La schizophrénie, Idées reçues, Editions Le cavalier bleu, Paris.2006, P15.

(4) Monestés Jean-Louis, La schizophrénie, Editions 0dile Jacob, Paris.2008, P19.

(5) Minkowski Eugene, La schizophrénie, Editions Payot, Paris.2002, P8.

(6) Franck Nicolas, La schizophrénie, La reconnaître et la soigner, Editions Odile Jacob, Paris 2006, PP.18, 19.

(7) Monestés Jean-Louis, Op-cit, PP.21,22.

(8) Gourion Dauid et Gut-Fayand Anne, Op-cit, P31.

(9) Pankow Giséla, L'être - là du schizophrène, 2eme Editions Aubier Montaigne, paris, 2011, P.54.

(10) Monestés Jean-Louis, Op-cit. P.24.

(11) KingDon David.C et Turkin Gton Douglas, Thérapie cognitive de la schizophrénie, Editions De Boeck université, Paris2011, P.56.

(12) Monestés, Jean-Louis, Op-cit, P.24.

(13) Mranck Nocolas, Op-cit, P.P.P.23.25.28.30. 
(14) Gourion David et Gut-Fayand, Op-cit, PP.17.18.

(15) Monestes Jean-Louis, Op-cit, P.29

(16) Gourion David et Gut-Fayand, Op-cit,P.44.

(17) Monestes Jean-Louis, Op-cit, P.30.

(18) Granger Bernard, Naudir Jean, Op-cit, P.44.

(19) Franck Nicolas, Op-cit, P.P.99.100.

(20) ${ }^{1}$ Grunberg Lalonde, Psychiatrie clinique - approche bio-psychosociale, Editions ESKA S.A.R.L, Canada, 1988, P.324.

(21) Monestes Jean-Louis, Op-cit, P.35.

(22) Gourion David et Gut-Fayand, Op-cit,P.P.27.28.

(23) Granger Bernard et Naudin Jean, Op-cit, P.81. 
العدد 01 مارس 2012 